\title{
Adaptive Processing Gain Data Services in Cellular CDMA in Presence of Soft Handoff with Truncated ARQ
}

\author{
Dipta DAS (Chaudhuri)", Sumit KUNDU ${ }^{+}$ \\ *Department of Electronics \& Communication Engg, Dr. B. C. Roy Engg College, Durgapur, India \\ ${ }^{+}$Department of Electronics \& Communication Engg, National Institute of Technology, Durgapur, India \\ Email:dipta_chaudhuri07@rediffmail.com,sumit.kundu@nitdgp.ac.in \\ Received October 27, 2008; revised December 8, 2008; accepted February 11, 2009
}

\begin{abstract}
An adaptive data transmission scheme based on variable spreading gain (VSG) is studied in cellular CDMA network in presence of soft handoff (HO). The processing gain is varied according to traffic intensity meeting a requirement on data bit error rate (BER). The overall performance improvement due to processing gain adaptation and soft $\mathrm{HO}$ is evaluated and compared with a fixed rate system. The influence of soft HO parameters on rate adaptation and throughput and delay performance of data is indicated. Further truncated automatic repeat request (T-ARQ) is used in link layer to improve the performance of delay sensitive services. The joint impact of VSG based transmission in presence of soft handoff at physical layer and T-ARQ at link layer is evaluated. A variable packet size scheme is also studied to meet a constraint on packet loss.
\end{abstract}

Keywords: Adaptive Systems, Variable Spreading Gain, Soft Handoff, ARQ

\section{Introduction}

Multimedia services are becoming increasingly important in wireless networks. The demand for high rate packet data transmission and quality of services (QoS) in wireless networks is growing at a rapid pace. Code Division Multiple Access (CDMA) is very promising to meet the demand for high data rate and quality of service (QoS) in wireless networks. The cellular capacity of CDMA system is limited in the uplink by maximum tolerable interference at the base station (BS). The cellular capacity indicates that there is a practical number of admissible users which should not be exceeded in order to ensure QoS (quality of service) of the admitted users. However for a fixed data rate system, there will be redundant margin on system capacity when traffic level is much lower than the maximum allowed number. Adaptive transmission schemes utilize the system resource more efficiently over a fixed rate scheme where the data transmission rate is controlled depending on generated interference or channel traffic intensity [1-3]. Packet data transmission is gaining importance in CDMA networks. Variable processing gain (VSG) and multi-codes
(MC) are two interesting approaches for increasing transmission rate of data in CDMA [4].

CDMA uses soft handoff (HO) where the handoff mobile near a cell boundary transmits to and receives from two or more BS-s simultaneously [5]. Soft HO provides a seamless connectivity, reduces "ping-pong" effect as present in hard HO, lowers probability of lost calls and eases power control [5,6]. It extends the coverage and increases the reverse link capacity $[6,7]$ by reducing overall interference.

Several research papers have analyzed adaptive transmissions based on VSG [1-4] without considering the effects of soft HO. Since soft HO affects the generated interference, it is expected to have significant impact on spreading gain selection and successful transmission of packet data, which is considered in the present paper.

However performance of data services is limited by interference and channel fading. Adaptive modulation and coding (AMC), adaptive antenna array providing space diversity and several receiver algorithms at physical layer $[8,9]$ are used to enhance the throughput performance of packet data oriented systems. Alternately 
channel fading can be mitigated by automatic repeat request (ARQ) protocol at the data link layer which ensures persistent retransmission of packets associated with a particular message until it is received correctly. ARQ is effective in improving system throughput relative to only forward error correction (FEC) [9]. Further ARQ can be combined with FEC in a hybrid ARQ scheme. To minimize delay and buffer sizes in practice, truncated ARQ has been adapted to limit the number of retransmissions. Further the transmission of video/image requires the delay to be bounded i.e. a packet is to be dropped if it is not received correctly after a finite number of retransmissions [8].

Several research papers have studied the combined effects of physical layer issues with the link layer issues like ARQ [8,9]. However the issue of soft handoff at physical layer is not considered in $[8,9]$.

In the present paper we consider VSG based data transmission meeting a constraint on upper limit of BER in presence of soft handoff. A simulation study is carried out to evaluate performance of packet data in terms of throughput and delay considering the joint effects of VSG and soft handoff. Two cases of retransmissions namely infinite ARQ and truncated ARQ at link layer are also considered. First the performance of data has been simulated in VSG with soft HO considering infinite ARQ. Next T-ARQ is considered at link layer for real time services along with soft $\mathrm{HO}$ at physical layer. Joint effects of soft $\mathrm{HO}$ and truncated ARQ on throughput and delay performance of a packetized data are evaluated for an imperfect power control CDMA. The performance in each case is compared with fixed rate. Effects of soft HO parameters on spreading gain adaptation and data performance in terms of throughput, delay and packet loss associated with truncation are indicated. Further a variable size packet transmission is also considered to meet a constraint on packet loss rate. Thus it has been possible to satisfy the BER constraint, delay constraint and the packet level QoS such as packet loss rate as well.

Sections 2 and 3 briefly describes the cellular scenario and our simulation model. Results and discussions are presented in Section 4. Finally we conclude in Section 5.

\section{System Model}

A cluster of three sectored cells with uniformly distributed mobile data users (MS) and equal number of MS-s $\left(N_{d}\right)$ per sector are considered. All data users transmit at the same rate using a single code. For fixed rate system the user transmits on single code at a fixed rate $R_{b}$ while in adaptive system the transmission rate $R_{b}(l)$ is variable depending on traffic load $(l)$ which is Poisson distributed with mean $(\lambda)$. The processing gain $(p g)$ of all codes are equal; where $p g(l)=\mathrm{W} / R_{b}(l)$; W is spread bandwidth. Processing gain $p g(l)$ (hence data rate $R_{b}(l)$ ) is selected depending on traffic load (l) satisfying a BER criterion. A “continuously active” data traffic model as in [10] is considered where each user generates a sequence of fixed length packets. A new packet is generated as soon as the preceding packet is either delivered successfully or dropped due to truncation in ARQ. The soft HO region is defined based on the distance from the base station (BS) as in Figure 1. An MS located outside the handoff boundary $R_{h}$ is considered to be under soft $\mathrm{HO}$ with three neighboring BS-s. Each sector is divided into two regions, soft $\mathrm{HO}$ regions (B, C, D) and non-HO region (A, E, F) of cell \#0,1and 2 respectively in Figure $1 . \mathrm{BS}_{0}, \mathrm{BS}_{1}$ and $\mathrm{BS}_{2}$ are the $\mathrm{BS}$-s of cell \#0, 1 and 2 respectively. The propagation radio channel is modeled as in [7]. The link gain for a location

$$
(r, \theta) \text { is } G_{i}(r, \theta)=d_{i}(r, \theta)^{-\alpha_{p}} 10^{\xi_{S / 10}}
$$

where $d_{i}(r, \theta)$ is the distance between the MS and $B S_{i}, \alpha_{p}$ is the path loss exponent and $10^{\xi_{s} / 10}$ is the log-normal component with $\xi_{s}$ normally distributed with 0 mean and variance $\sigma_{s}^{2}$. The shadow fading at i-th BS is [7]

$$
\xi_{s-i}=a \zeta+b \zeta_{i} \text { with } a^{2}+b^{2}=1
$$

$\zeta$ and $\zeta_{i}$ are independent Gaussian random variables with zero mean and variance $\sigma_{s}^{2}$. Out-cell interference consists of interference due to MS-s from region (E,C,G,H) of cell \#1 and (D,F,I,J) of cell \#2. MS-s in furthest sectors $(\mathrm{G}, \mathrm{H}, \mathrm{I}, \mathrm{J})$ are assumed to be power controlled by respective BS-s. The reference user is located in non-HO region of reference sector i.e. in region ' $A$ '. Total in-cell interference in cell \# 0 is

$$
I_{\text {in }}=I_{1}+I_{2}
$$

where $I_{1}$ is due to all MS-s in A and those in B connected to $\mathrm{BS}_{0}, I_{2}$ is due to MS-s in B but connected to $\mathrm{BS}_{1}$ and $\mathrm{BS}_{2}$. The out-cell interference is

$$
I_{\text {out }}=2\left(I_{E}+I_{c 1}+I_{c 2}+I_{c o}+I_{G}+I_{H}\right)
$$

$I_{E}$ is the interference due to MS-s in E and connected to $\mathrm{BS}_{1}$. Similarly $I_{c 1}$ and $I_{c 2}$ are due to MS-s in region $\mathrm{C}$ and power controlled by $\mathrm{BS}_{1}$ and $\mathrm{BS}_{2}$ respectively. $I_{c o}$ is due to MS-s in $\mathrm{C}$ and controlled by $\mathrm{BS}_{0}$. $I_{G}$ and $I_{H}$ are the interference due to MS-s in G and H. MS-s in these farthest sectors are assumed to be power controlled by respective $\mathrm{BS}$ i.e. $\mathrm{BS}_{1}$. A multiplication factor of two is used in Equation (4) to include 
contribution of cell \#2. The actual received power from desired user is $U=S_{R} e^{S}$, where $\mathrm{S}$ is a Gaussian r.v. with mean 0 and variance $\sigma_{S}^{2}=\sigma_{e}^{2}$. The BER $\left(P_{e}\right)$ for data user is simulated as described in later section in the above soft HO environment considering direct sequence spreading and BPSK data modulation having spread b.w of $\mathrm{W}$. The maximum allowed bit rate of data users $R_{b}{ }^{*}(l)$ for traffic intensity is adjusted such that $\left(P_{e} \leq \beta\right)$, the corresponding processing gain is selected as $\mathrm{pg}^{*}(l)$. The retransmission probability $\mathrm{P}_{\mathrm{r}}$ is given as [11]

$$
P_{r}=1-\left(1-P_{e}\right)^{L_{p} r_{c}}
$$

where $\mathrm{L}_{\mathrm{p}}$ is the length of the packet in bits and $r_{c}$ is the FEC code rate. For continuously active data users, the average packet delay is the same as the packet transfer time $T_{p}$ as there is no waiting delay in the queue. The time required for transmitting a packet of length $L_{p}$ by a data user transmitting at a rate of $R_{b}(l)$ is :

$$
T_{p}=\frac{L_{p}}{R_{b}(l)}=\frac{L_{p} \quad p g^{*}(l)}{R_{c}}
$$

where $R_{c}$ is the chip rate. We assume that acknowledgement from the receiver is instantaneous and perfectly reliable. In case of truncated ARQ the maximum number of retransmissions in ARQ has to be bounded since only finite delay and buffer sizes can be afforded in practice. If $T_{\max }$ is the maximum allowed packet delay, the maximum number of retransmissions is given as $N_{\max }=\left\lfloor T_{\max } / T_{p}\right\rfloor$. Thus with truncated ARQ if a packet is not received correctly after $N_{\max }$ retransmissions, it is dropped and declared as a packet loss. The average delay with truncation

$$
D=\frac{L_{p} \cdot p g^{*}(l)}{R_{c}}\left\{\frac{1-P_{r}^{\left(N_{t}+1\right)}}{1-P_{r}}\right\}
$$

The average throughput is defined as the average number of information bits successfully transferred per sec and is given as

$$
G=\frac{L_{p} \cdot r_{c}}{D}=\frac{r_{c} \cdot R_{c}\left(1-P_{r}\right)}{p g^{*}(l)\left(1-P_{r}^{\left(N_{t}+1\right)}\right)}
$$

Some services may require maintaining packet loss (packet QoS) below a prescribed $\operatorname{limit}(\delta)$. A variable packet length scheme is used where the packet size is adjusted under different traffic and soft HO conditions so as to maintain packet loss below a desired limit $(\delta)$. The length of the packet $\left(L_{p}^{*}\right)$ is selected satisfying $P_{\text {loss }} \leq \delta$ :

$$
\text { i.e. }\left\{1-\left(1-P_{e}\right)^{L_{p}^{*} r_{c}}\right\}^{N_{t}^{*}+1} \leq \delta, \quad N_{t}^{*}=\left\lfloor T_{\max } / T_{p}^{*}\right\rfloor
$$

where $T_{p}^{*}=L_{p}^{*} / R_{b}$

The packet size $L_{p}^{*}$ is found by simultaneously satisfying (9) and (10).

However with infinite retransmission i.e. infinite ARQ, there is no packet loss. In such situation the average delay [11]

$$
\mathrm{D}=\frac{T_{i}}{\left(1-P_{r}\right)}=\frac{L_{p} p g^{*}(l)}{R_{c}\left(1-P_{r}\right)}
$$

and the average throughput $(\mathrm{G})$ given as

$$
G=\frac{L_{p} r_{c}}{D}=\frac{r_{c} R_{c}\left(1-P_{r}\right)}{p g^{*}(l)}
$$

In the next section we present our simulation model for both infinite and truncated ARQ cases.

\section{Simulation Model}

The simulation is developed in MATLAB using the following parameters: $\mathrm{PR}_{\mathrm{h}}$ indicates the degree of soft $\mathrm{HO}$, shadowing correlation $\left(\mathrm{a}^{2}\right)$, pce $\sigma_{e}$, traffic intensity $\lambda$. he soft $\mathrm{HO}$ region boundary $R_{h}$ given as $R_{h}=R_{o} \sqrt{1-P R_{h}}$ where $R_{o}$ is the radius of the cell, normalized to unity and hexagonal cell is approximated by a circular one with radius $R_{o}$. Users are assumed to be uniformly distributed.

\subsection{Generation of Users Location and Interference}

1) The number of users $\left(N_{d}\right)$ is generated by generating a Poisson distributed r.v with mean $\lambda$.

2) Locations $(r, \theta)$ of all $\left(N_{d}\right)$ users are generated and users are divided into non-HO $\left(N_{h}\right)$ and soft $\mathrm{HO}$ $\left(N_{s}\right)$ region based on their location. Assuming the desired user in non-HO region, let the remaining interfering users in non-HO are $\left(N_{h}-1\right)$. Number of users in soft HO region: $N_{s}=N_{d}-N_{h}$.

3) For each of those in soft HO region $\left(N_{s}\right)$, the link gains corresponding to each of three BS-s involved in soft $\mathrm{HO}$ are generated as

$G_{i}(r, \theta)=r_{i}^{-\alpha_{p}} e^{\xi_{i}}, \mathrm{i}=0,1,2$. where $\xi_{i}$ is a Gaussian r.v with mean 0 and variance $b^{2} \sigma_{s}{ }^{2}, r_{i}$ is the distance from i-th BS. The user is power controlled by the BS for which the link gain is maximum i.e. it is power controlled by $\mathrm{BS}_{\mathrm{i}}$ if $G_{i}$ is maximum; $i=0,1,2$. 
4) The interference received at reference BS

$$
\mathrm{I}=S_{R} \exp \left(r_{n}\right)\left(\frac{G_{0}}{G_{i}}\right)
$$

if connected to $\mathrm{BS}_{\mathrm{i}}$, where $i=0,1,2$. Here $r_{n}$ is a normal r.v. with 0 mean and standard deviation $\sigma_{e}$. $S_{R}$ is the required received power at the respective BS which is normalized to unity in the simulation since SIR is unaffected by assigning $S_{R}=1$.

5) Next interference due to $\left(N_{h}-1\right)$ MS-s in non_HO region (A) of reference cell, each power controlled by $\mathrm{BS}_{0}$ is considered as

$$
I_{2}=S_{R} \sum_{i=1}^{N_{h}-1} e^{r_{n}, i}
$$

6) Now the interference due to MS-s in adjacent sectors i.e. (region E,C,D and F) of cell\#1 and \#2 are found in similar manner. The number of MS-s in $\mathrm{E}$ and $\mathrm{F}$ are $\left(N_{d}-N_{s}\right)$ each. Let $I_{3}=I_{E}+I_{C}$ and $I_{4}=I_{D}+I_{F}$

7) Interference from MS-s in G,H, I and J regions are generated following step 4. Let $I_{5}=I_{G}+I_{H}$ and $I_{6}=I_{I}+I_{J}$.

8)Total interference $\mathrm{I}=\sum_{k=1}^{6} I_{k}$

9) Signal from desired user

$$
U=S_{d} e^{x}, S I R=U / I
$$

$x$ is Gaussian with mean ' 0 ' and variance $\sigma_{e}^{2}$

\subsection{BER Simulation}

A Gaussian noise sample $n_{g}$ with ' 0 ' mean and variance $\sigma_{g}^{2}=1 . /(2 \mathrm{pg} . \mathrm{SIR})$ is added to each bit of a transmitted sequence and received bits are compared with the transmitted bits. Here SIR is found following steps A(1) to A(8) for a given $p g$.

\subsection{Selection of Processing Gain}

1. An initial low value of $R_{b}$ is chosen and BER is simulated as described above.

2. $R_{b}$ is incremented in steps, the highest value of

$R_{b}{ }^{*}$ for which $B E R \leq \delta$, is chosen and corresponding processing gain $p g^{*}=\left\lceil W / R_{b}^{*}\right\rceil$ is selected.

\subsection{Packet Loss and Variable Packet Length}

1) For truncated $A R Q$ case:

A sample of Gaussian noise as in (B) is added to each transmitted bits of a packet of $L\left(=L_{p} r_{c}\right)$ information bits. The received L bits of a packet are checked with their corresponding transmitted bits to assess packet error. If the received packet is incorrect, the same packet (i.e. the same bit pattern) is re-transmitted $N_{t}$ times, where $N_{t}=$ $1,2, \ldots N_{\max }$. A packet loss occurs if it is not received correctly after $N_{t}$ re-transmissions. Average delay (D) is estimated as: $\left(\left(N_{p}+\right.\right.$ retx $x_{-}$count $\left.) . / N_{p}\right) T_{p}$ where $T_{p}$ is as in (6), $N_{p}$ : number of transmitted packets, retx_count : total retransmissions of $N_{p}$ packets (each packet is retransmitted maximum up to $N_{\max }$ time.

The throughput is: $G=L_{p} r_{c} / D$

An initial small packet size is chosen and it is incremented in steps $(\Delta=2)$ till packet loss just exceeds $\delta$. The highest packet length for which $P_{\text {loss }}<\delta$ is $L_{p}^{*}$. Now throughput, delay and packet loss are estimated by simulation following steps as mentioned above where $p g$ is chosen as $p g^{*}(l)$ and $L_{p}$ is chosen as $L_{p}^{*}$.

2) For infinite ARQ case:

If the received packet is incorrect, the same packet is retransmitted until the packet is finally received correctly instead of limiting the retransmission to $N_{\max }$ as in case of T-ARQ. Then delay and throughput in this case are estimated in the same manner as described above. Total number of erroneous packet is counted out of a large number of transmitted packets to estimate the packet error rate (PER).

\section{Results and Discussions}

The parameters assumed in simulation are listed in Table 1 as shown. For the case of truncation we assume $T_{\max }=350 \mathrm{msec}$ and $N_{t}=\left\lceil N_{\max } / 2\right\rceil$ where $N_{\max }$ is maximum retransmission corresponding to $T_{\max }$.

Table 1. Parameters for simulation.

\begin{tabular}{cccccc}
\hline $\begin{array}{c}\text { Spread } \\
\text { BW }(\mathrm{W})\end{array}$ & $\begin{array}{c}\text { Chip rate } \\
R_{c}\end{array}$ & $\begin{array}{c}\text { Fixed } \\
\text { PG }\end{array}$ & $\begin{array}{c}\text { Fixed } \\
L_{p}\end{array}$ & $\begin{array}{c}\text { SIR thresh- } \\
\text { old } \gamma_{t h}\end{array}$ & $\begin{array}{c}\text { pce } \\
\sigma_{e}(\mathrm{~dB})\end{array}$ \\
\hline $5.0 \mathrm{MHz}$ & $5.0 \mathrm{Mcps}$ & 312 & $\begin{array}{c}1024 \\
\text { Shadowing } \\
\text { correlation } \\
\left(a^{2}\right)\end{array}$ & $\begin{array}{c}\text { Degree of } \\
\text { soft HO } \\
P R_{h}\end{array}$ & $\begin{array}{c}\text { Max al- } \\
\text { lowed } \\
\text { packet loss }\end{array}$ \\
$\alpha_{p}$ & $\sigma_{s}(\mathrm{~dB})$ & $r_{c}$ & $\begin{array}{c}\text { and } 2 \\
\text { and } 0.3\end{array}$ & $0.3,0.7$ & $5 \%$ \\
4 & 6 & 0.5 & & & \\
$\begin{array}{c}\text { Target BER } \\
10^{-3}\end{array}$ & $\begin{array}{c}T_{\text {max }} \\
\text { 350msec }\end{array}$ & & & & \\
\hline
\end{tabular}




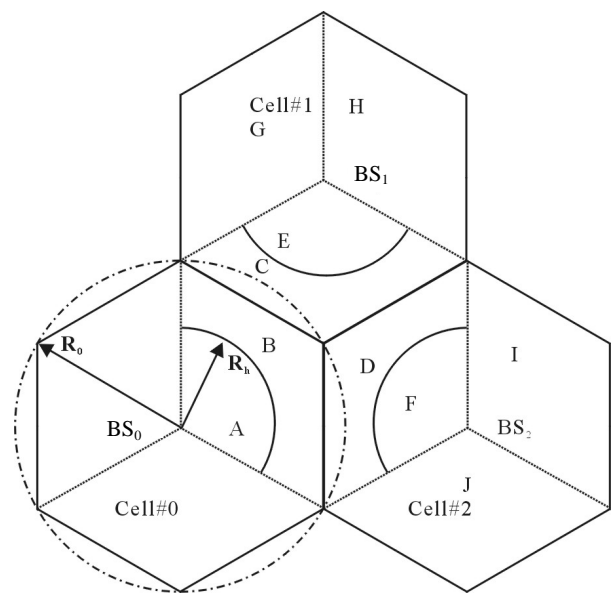

Figure 1. Cellular Layout for soft HO. A, E, F are non HO region. $\mathrm{B}, \mathrm{C}, \mathrm{D}$ are soft $\mathrm{HO}$ region. Cell \# $\mathrm{O}$ is reference cell.

Figure 2 shows the effects of soft HO on BER performance of fixed rate and adaptive transmission. In adaptive transmission, the BER is always maintained as $P_{e} \leq 10^{-3}$ for the entire range of traffic by adjusting the $p g$ i.e. transmission rate is reduced with increase in traffic as in curves (iv,v). While in fixed rate ( $\mathrm{PG}=312)$, the BER increases with increase in traffic intensity due to increased interference as in curves (i, ii and iii). Higher degree of soft $\mathrm{HO}$ reduces BER in fixed $p g$ case as seen in curves (i) and (ii) due to reduced level of interference. In case of adaptive transmission, as the transmission rate is adjusted (varying the pg) keeping BER fixed, a higher degree of soft HO will lead to increase in data rate i.e. lower processing gain allocation for same BER constraint of $P_{e} \leq 10^{-3}$. Two cases of adaptive pg with different levels of soft $\mathrm{HO}$ have been shown in curves (iv, v) where BER $\leq 10^{-3}$ in both the cases. However the data rate (allocated pg) for these cases will be significantly different as seen in next fig.

Figure 3 shows the effects of soft $\mathrm{HO}$ on allocation of processing gains in VSG based adaptive transmissions. The processing gains $(p g)$ are chosen satisfying BER $\leq 10^{-3}$ under different conditions of soft HO. As the traffic intensity $(\lambda)$ increases, data rate is reduced by increasing pg so as to reduce the interference level for maintaining BER constraint. Higher degree of soft HO reduces BER for fixed data rate. As BER is kept fixed, higher degree of soft $\mathrm{HO}$ will allow higher data rate or lower value of allocated $p g$ in curves (ii and iii). Thus it is seen that as $P R_{h}$ increases from 0.3 to 0.7 , allocated $p g$ for $\mathrm{BER} \leq 10^{-3}$ reduces from 339 to 260 at $\lambda=8$. Higher shadowing correlation $\left(a^{2}\right)$ as well as lower pce also reduces BER for fixed PG. Thus in a similar manner as in case of $P R_{h}$, this will also increase data rate (or reduce processing gain) for a target BER as seen in curves (i,iii) and curves (iii,iv).

The throughput performance with T-ARQ is depicted in Figure 4. Adaptive pg based transmission is seen to achieve a higher throughput as compared to a fixed one for most range of traffic in curves (ii,iii). This is because BER is maintained below a limit in adaptive case whereas it increases with traffic in case of fixed pg. Further improvement in throughput is achieved with higher degree of soft handoff in curves (i,ii). However as seen in Figure 4 vide curves (ii,iii), over a range of traffic say up to $\lambda=7$, adaptive pg and fixed pg yields close throughput performance. This is because our arbitrary chosen value of fixed pg i.e. $\mathrm{PG}=312$ is close to adaptive pg-s found (satisfying $P_{e} \leq 10^{-3}$ ) over this traffic range. For example at $\lambda=7$, allocated adaptive $p g=304$.

Effects of soft HO parameters on packet delay are depicted in Figure 5. Truncation always maintains packet delay below a certain level, here 0.5 times of $T_{\max }=350$ msec, as number of retransmission $N_{t}=\left\lceil N_{\max } / 2\right\rceil$. Adaptive transmission yields less delay as compared to fixed case for most range of traffic. Adaptive transmission always maintains a fixed BER hence a fixed level of PER (packet error rate) while BER increases with traffic in case of fixed pg. However around $\lambda=7$ the performance of fixed and adaptive are close as our chosen fixed pg of 312 becomes close to found value of adaptive pg. Higher degree of soft handoff is found to lower delay further as seen in curves (ii,iii).

Figure 6 shows the packet loss associated with T-ARQ vs traffic intensity for fixed and adaptive pg based transmissions. Adaptive pg always maintains a fixed level of packet error. Thus adaptive pg yields lower packet loss as compared to fixed rate for moderate traffic in curves (i,ii). The packet loss reaches a floor for adaptive case while it increases with higher traffic in case of fixed rate. Further the packet loss is kept below $\delta=5 \%$ using variable packet size as seen in curve (iv). Higher soft handoff reduces packet loss as in curves (ii, iii).

Figure 7 shows the variation of packet size with traffic intensity. The packet size is varied in order to ensure that packet loss is always $\leq 5 \%$. Higher traffic intensity requires smaller size of packet. Further higher degree of soft handoff $\left(P R_{b}\right)$ can transmit larger packet as seen in curves (i,iii). Higher shadowing correlation also allows larger packet while meeting the loss constraint as in curves (i,ii).

Table 2 shows the maximum number of retransmissions allowed vs traffic under two different soft handoff conditions. As the traffic intensity increases, the allo- 
Table 2. Maximum number of retransmissions vs traffic (mean arrival rate) for adaptive pg based transmissions.

\begin{tabular}{ccccccccc}
\hline$\lambda$ & 5 & 6 & 7 & 8 & 9 & 10 & 11 & 12 \\
\hline (1) $N_{\max }$ & 6 & 5 & 4 & 4 & 3 & 3 & 2 & 2 \\
(2) $N_{\max }$ & 8 & 7 & 6 & 5 & 4 & 4 & 4 & 3 \\
\hline
\end{tabular}

(1) $a^{2}=0.3, P R_{h}=0.3$, pce $=2 d B \quad T_{\max }=350 \mathrm{msec}$

(2) $a^{2}=0.3, P R_{h}=0.7, p c e=2 d B \quad T_{\max }=350 \mathrm{msec}$

cated data rate is reduced (i.e. allocated pg increases). Thus the packet transfer time is increased which in turn reduces the maximum number of retransmissions for a prescribed maximum allowed delay. Higher soft HO allows more number of retransmissions.

Figure 8 shows the effects of truncated ARQ on delay. We have chosen truncation in retransmission as $N_{t}=\left\lceil N_{\max } / 2\right\rceil$ to ensure a maximum delay of 0.5 times of $T_{\max }=350 \mathrm{msec}$ i.e. maximum allowed delay of 175 msec in this case. It is seen that in case of infinite re-

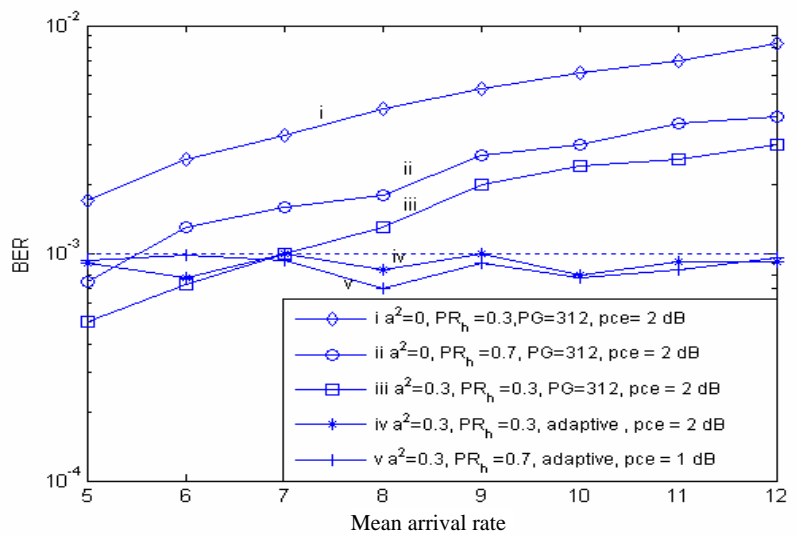

Figure 2. BER vs mean arrival rate ( $\lambda$ users/sec) per sector.

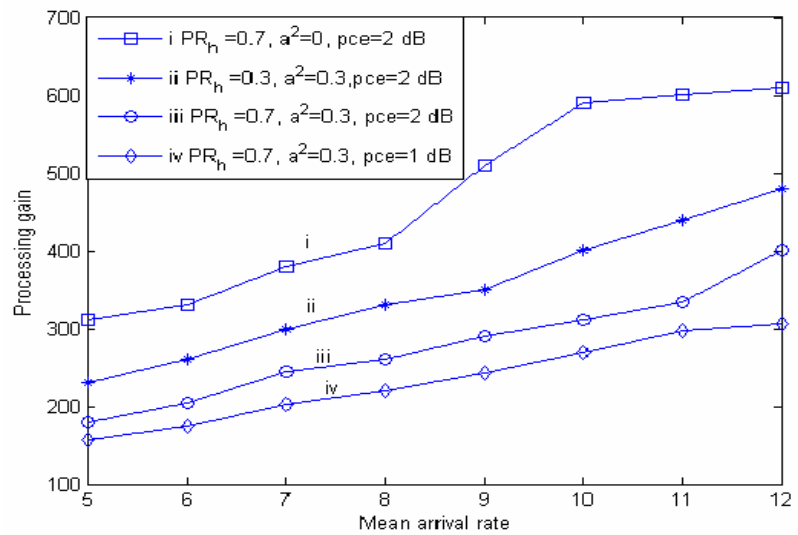

Figure 3. Processing gain vs mean arrival rate ( $\lambda$ users/ sec) per sector. transmission, the delay increases rapidly with traffic intensity. T-ARQ always yields lower delay as compared to infinite ARQ (curves ii,iii). Further in case of T-ARQ, though delay increases with traffic, it is always maintained below a chosen desired limit of 175 msec (i.e. 0.5

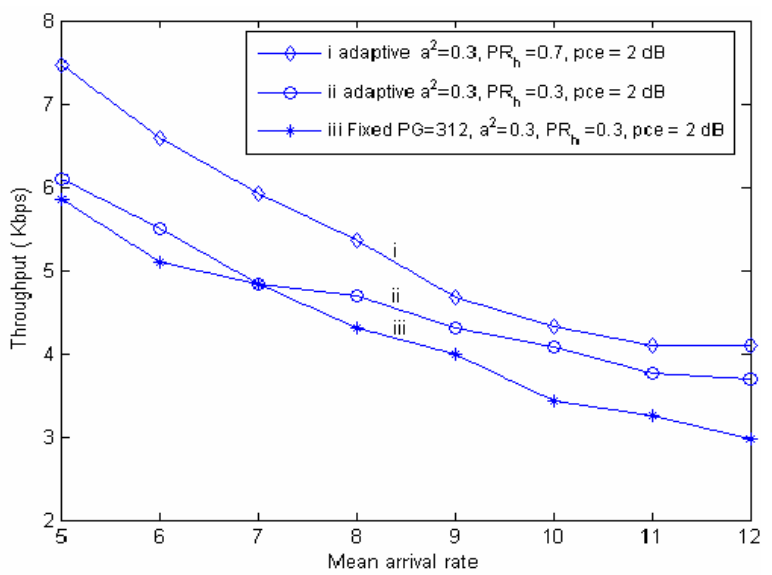

Figure 4. Throughput (Kbps) vs mean arrival rate.

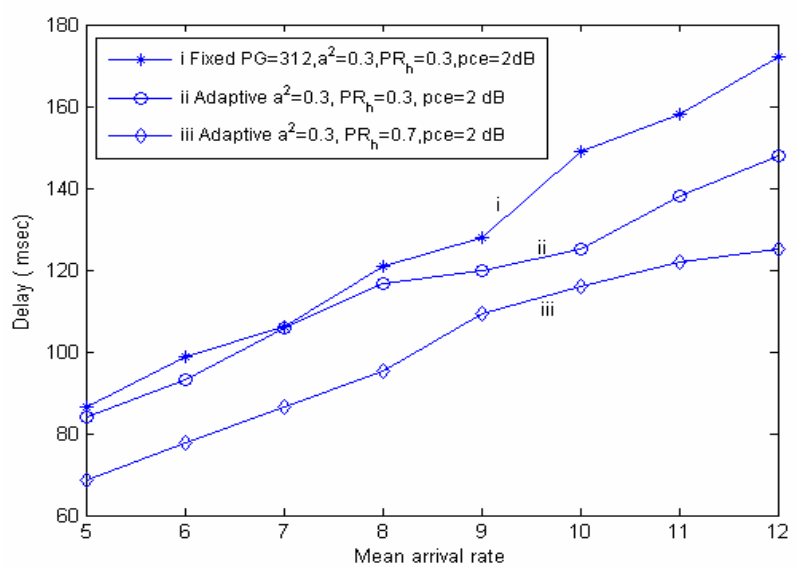

Figure 5. Delay (msec) vs mean arrival rate.

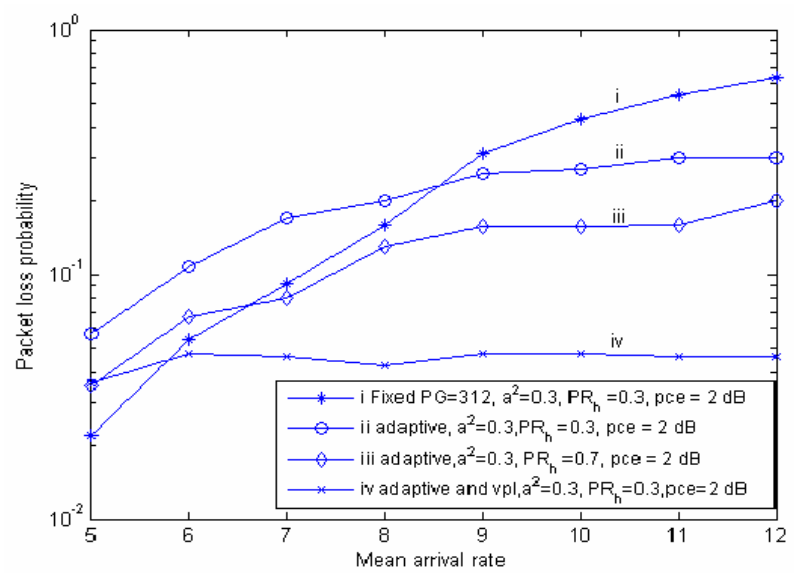

Figure 6. Packet loss probability vs mean arrival rate. 
$\left.T_{\max }\right)$. With infinite retransmission the delay exceeds desired limit of $175 \mathrm{msec}$ for $\lambda=10$ onwards in case of $\mathrm{a}^{2}=0.3$ and $\lambda=6$ onwards for $\mathrm{a}^{2}=0$ in curves (i,ii). Thus T-ARQ satisfies delay constraint at the cost of packet loss. Further higher shadowing correlation lowers the delay in curves (i,ii).

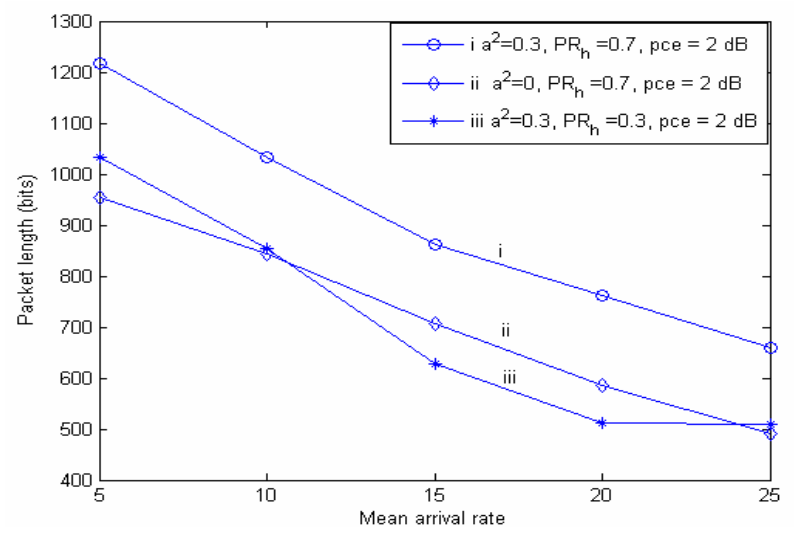

Figure 7. Effects of soft $\mathrm{HO}$ and shadowing correlation on packet length for adaptive pg based Transmissions.

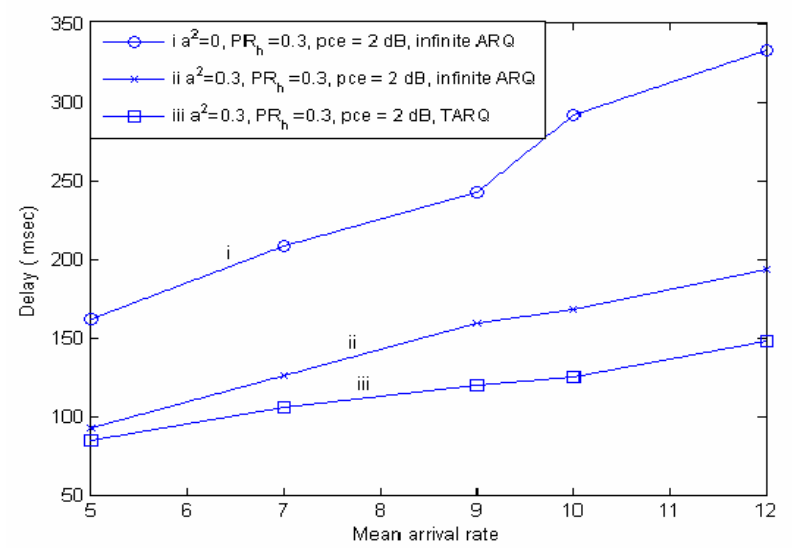

Figure 8. Effects of truncation in ARQ on delay.

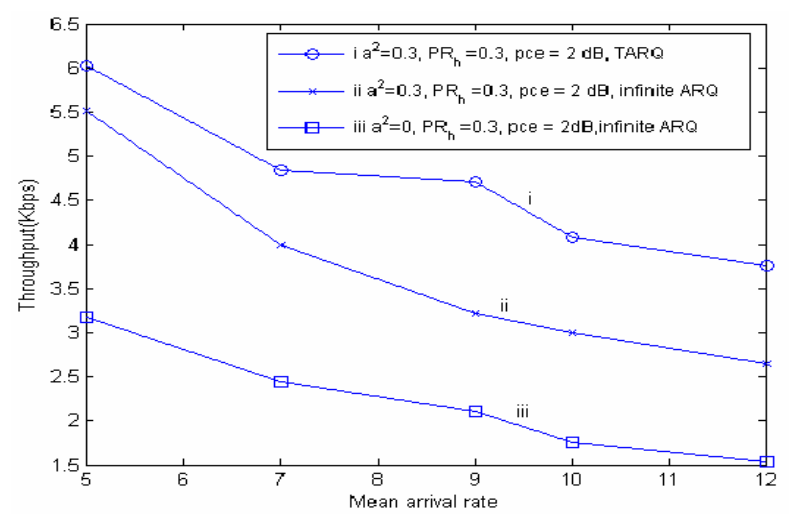

Figure 9. Effects of truncation in ARQ on throughput.
Figure 9 shows the effects of truncated ARQ and shadowing correlation on throughput. In present work throughput indicates the average number of information bits successfully transferred per sec. Since a packet is delivered successfully with in a given time limit in truncation (or dropped if not successful), truncation yields higher throughput as compared to infinite retransmissions as in curves (i,ii). Also higher shadowing correlation improves throughput as in curves (ii,iii).

\section{Conclusions}

Performance of packet data is evaluated in presence of soft handoff under adaptive transmission based on variable processing gain and is compared with a fixed rate system. The processing gain is varied according to traffic intensity so as to ensure an upper limit on channel BER. Truncated ARQ is used to ensure a prescribed maximum limit on packet delay. Soft handoff parameters, shadowing correlation, pce and T-ARQ are found to have significant impact on processing gain allocation and data performance. Adaptive transmission outperforms fixed transmission in terms of throughput, delay and packet loss. Adaptive transmission enhances throughput by $19 \%$ and reduces packet loss by $16 \%$ for a traffic intensity of $\lambda=10$ users/sec under a given soft handoff scenario. Higher degree of soft handoff and higher shadowing correlation improve the situation further. An increase in degree of soft handoff by $133 \%$ further enhances throughput by $6 \%$ and reduces packet loss by $12 \%$ for a traffic intensity of $\lambda=10$ users/sec. Using a variable packet length transmission the packet loss could be maintained below a limit. Thus data BER, delay constraint and packet QoS such as packet loss are simultaneously satisfied using variable processing gain, variable packet length and T-ARQ. Higher degree of soft handoff and higher shadowing correlation allow transmission of larger packet size. An increase in degree of soft handoff by $133 \%$ allows $20 \%$ increase in packet size for a traffic of $\lambda=10$ users/sec satisfying a packet loss constraint.

\section{References}

[1] K. Choi, S. Kim, Shin, and K. Cheun, "Adaptive processing gain CDMA networks over poison traffic channel,” IEEE Communications Letters, Vol. 6, No. 7, pp. 273-75, July 2002.

[2] K. Choi, Y. Chae, and J. Park, “Throughput-delay performance of interference level adaptive transmission in voice/data integrated CDMA network with variable spreading gain,” IEE Proceedings on Communications, Vol. 151, No. 3, pp. 217-220, June 2004. 
[3] L. L. Yang and L. Hanzo, "Adaptive rate DS-CDMA systems using variable spreading factors," IEEE Transactions on Vehicular Technology, Vol. 53, No. 1, pp. 72-81, January 2004.

[4] S. Kumar and S. Nanda, "High data rate packet communication for cellular network using CDMA: Algorithms and performance,” IEEE Journal on Selected Areas in Communications, Vol. 17, No. 3, pp. 472-491, March 1999.

[5] D. Wong and T. Lim, "Soft handoff in CDMA mobile system,” IEEE Personal Communications, pp. 6-17, December 1997.

[6] H. Jiang and C. H. Davis, "Coverage expansion and capacity improvement from soft handoff for cellular CDMA," IEEE Transactions on Wireless Communications, Vol. 4, No. 5, pp. 2163-2171, September 2005.

[7] J. Y. Kim and G. L. Stuber, "CDMA soft HO analysis in the presence of power control error and shadowing correlation,” IEEE Trans on wireless Communications, Vol. 1,
No. 2, pp. 245-255, April 2002.

[8] Q. Liu, S. Zhou, and G. B. Giannakis, "Cross layer combining of adaptive modulation and coding with truncated ARQ over wireless links," IEEE Transactions on Wireless Communications, Vol. 3, No. 5, pp. 1746-1755, September 2004.

[9] B. Lu, X. Wang, and J. Zhang, “Throughput of CDMA data networks with multi-user detection, ARQ and packet combining,” IEEE Transactions on Wireless Communications, Vol. 3, No. 5, pp. 1576-1589, September 2004.

[10] J. Kim and M. Honig ,"Resource allocation for multiple class of DS-CDMA traffic,” IEEE Transactions on Vehicular Technology, Vo. 149, No. 2, pp. 506-518, March 2000.

[11] S. Kundu and S. Chakrabarti, "Performance of high rate data in wideband CDMA with correlated interferers," in GESTS International Transactions on Communication \& Signal Processing, Vol. 7, No. 1, pp. 53-64, June 2006. 